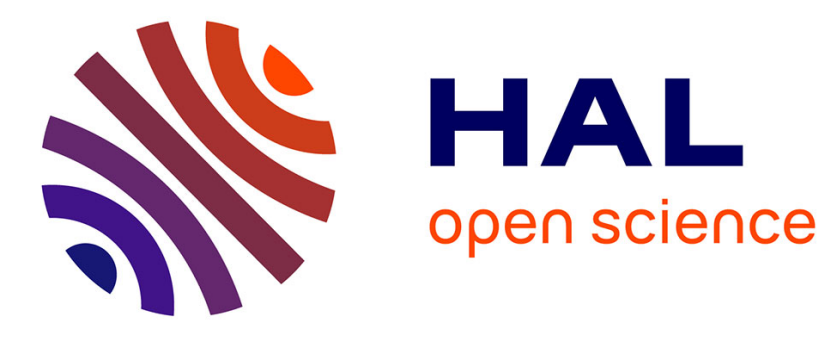

\title{
Delamination influence on elastic properties of laminated composites
}

\author{
Humberto Brito-Santana, Bruno Guilherme Christoff, Antonio Joaquim \\ Mendes Ferreira, Frédéric Lebon, Reinaldo Rodríguez-Ramos, Volnei Tita
}

\section{- To cite this version:}

Humberto Brito-Santana, Bruno Guilherme Christoff, Antonio Joaquim Mendes Ferreira, Frédéric Lebon, Reinaldo Rodríguez-Ramos, et al.. Delamination influence on elastic properties of laminated composites. Acta Mechanica, 2019, 230 (3), pp.821-837. 10.1007/s00707-018-2319-8 . hal-02335897

\section{HAL Id: hal-02335897 https://hal.science/hal-02335897}

Submitted on 21 Nov 2019

HAL is a multi-disciplinary open access archive for the deposit and dissemination of scientific research documents, whether they are published or not. The documents may come from teaching and research institutions in France or abroad, or from public or private research centers.
L'archive ouverte pluridisciplinaire HAL, est destinée au dépôt et à la diffusion de documents scientifiques de niveau recherche, publiés ou non, émanant des établissements d'enseignement et de recherche français ou étrangers, des laboratoires publics ou privés. 


\title{
Humberto Brito-Santana • Bruno Guilherme Christoff • Antonio Joaquim Mendes Ferreira • Frédéric Lebon • Reinaldo Rodríguez-Ramos • Volnei Tita \\ Delamination influence on elastic properties of laminated composites
}

\begin{abstract}
The present work aims to predict the behavior of effective elastic properties for laminated composites, considering localized damage in the interface between two layers. In practical terms, the damage in the adhesion, which influences the effective elastic properties of a laminate, is evaluated like a delamination between adjacent layers. Thus, the effective properties of laminated composites with different delamination extensions are calculated via finite element method and two-scale asymptotic homogenization method. It is investigated how the properties of the laminated composites are affected by the delamination extension and the thickness of the interface between layers. It is possible to conclude that the effective coefficient values decrease as the damage extension increases due to the fact that the delamination area increases. Besides, for all effective coefficients, except the effective coefficients $C_{12}^{*}, C_{13}^{*}$, and $C_{23}^{*}$, in the case without delamination, the coefficients decrease as the adhesive region thickness increases, and almost all coefficients decrease for complete separation of the interface. Numerical and analytical results are compared in order to show the potentialities and limitations of the proposed approaches. Finally, a numerical approach is used to simulate a specific case, where the interface is considered a functionally graded material.
\end{abstract}

\section{Introduction}

Composites usage has increased in various structural applications, because they offer significant weight savings and have shown attractive mechanical properties. Like metals, these materials are notch sensitive and lose much of their structural integrity when damaged. In addition, it is well known that interfaces play an important role in the mechanical behavior of advanced materials, such as laminated composites. Interface cracking, also known as interlaminar debonding or delamination [1], is one of the most commonly encountered failure modes in composite laminates and can cause severe performance and safety problems, such as stiffness and load bearing

H. Brito-Santana $(\varangle) \cdot$ B. G. Christoff · V. Tita

Department of Aeronautical Engineering, São Carlos School of Engineering, University of São Paulo, Av. João Dagnone 1100,

São Carlos, SP, Brazil

Telephone: $+55-16-997159456$

E-mail: hbritosantana@gmail.com

\author{
A. J. M. Ferreira \\ Mechanical Engineering Department, Faculty of Engineering of University of Porto, University of Porto, Porto, Portugal \\ F. Lebon \\ CNRS, Centrale Marseille, Laboratoire de Mécanique et d'Acoustique, Aix-Marseille Université, 4 Impasse Nikola Tesla, \\ CS 40006, 13453 Marseille Cedex 13, France \\ R. Rodríguez-Ramos \\ Faculty of Mathematics and Computing Sciences, University of Havana, San Lázaro esq. L, Vedado, CP 10400 Havana, Cuba
}


capacity reduction and even structural collapse. In particular, delamination or debonding at imperfect interfaces between the layers and interior cracks in the individual layers can occur either separately or simultaneously.

The increasing use of laminated composites in different branches of engineering has stimulated advances in the analysis of delaminations. The bonding state at the interfaces between two adjacent layers clearly plays a critical role in determining the mechanical behavior of a composite material. On the one hand, the effect of localized damage has been focused on micromechanical investigations, where the damage in the interface between fiber and matrix has been included in the micromechanical analysis of the repeating unit cell. Many researchers have published in this field [2-7]. In addition, there are many scientific contributions, experimental, numerical and analytical, which approach delamination problems in laminated composites, and they are mostly related to macroscale analyses in order to explain the mechanical behavior of the structure. This can be observed in [8-13], where the authors presented experiments and/or three-dimensional finite element models for static and dynamic analyses of internal delamination in multilayer composites. Delamination buckling and growth in slender composite laminates using a FEM based on the cohesive element were investigated successfully, as well $[14,15]$. Then, a method to predict the initiation of delamination in shell-like structures accounting for the anisotropic material was developed considering a solid-shell element. Other researchers [16] have focused on the delaminated shell panel model using two higher-order mid-plane kinematics and solved numerically via an FE (finite element) iterative process. The effect of using higher-order shape functions for computing traction forces was also studied by employing higher-order isogeometric interface elements [17]. Some analytical and semi-analytical approaches have focused on the prediction of debonding initiation between cement-based overlay and old concrete substrate under monotonous mechanical loading [18]. Additional analytical investigations can be found in the literature as the calculation of delamination of z-pinned composites using a non-dimensional analytical model [19] and a semi-analytical solution for the free vibration analysis of generally laminated composite Timoshenko beams [20], as well as a delamination analysis of laminated composites during drilling operation $[21,22]$. On the other hand, only few works that are related to macroscale analyses focus on determining effective properties when delamination takes place in laminated composites. In other words, macro-mechanical investigations are rarely found in the literature for the determination of effective properties and how the damage in the interface between two plies influences the mechanical behavior of a laminated composite.

Regarding the aspects mentioned above, the present work consists of predicting effective elastic properties for laminated composites in macroscale level, evaluating the influence of delamination. The novelty of this contribution is that the effective properties of laminated composites with localized delamination are calculated via AHM (with two-scale asymptotic homogenization method) and via an in-house finite element software written in Julia language. In fact, the present work is a continuation of a paper previously published by the authors [23], which was an investigation of debonding between two isotropic materials by using AHM and a commercial finite element software. Thus, in the present paper, four different stacking sequences of laminated composites are evaluated $\left([0]_{n},[0 / 90]_{n},[30 /-30]_{n}\right.$ and $[45 /-45]_{n}$ ) with an epoxy layer in the interface of the plies, considering different values for the interface thickness between layers and for different delamination extensions. A three-layer composite material with perfect and imperfect conditions between the plies is proposed and evaluated for both approaches (numerical and analytical). A three-dimensional (3D) representative volume element (RVE) model is developed for the numerical approach to compute the effective properties of the laminate. For the analytical approach, the two-scale asymptotic homogenization method (AHM) provides analytical expressions of the effective properties for the laminate. In addition, the coefficients for different damage degrees (delamination extension) using the analytical approach are calculated averaging the results of limit cases: the complete separation of the interface and perfect bonding. Besides, the associated local problems are derived, and, consequently, the local functions are solved directly. Numerical and analytical results are compared in order to evaluate the effectiveness and accuracies of the proposed approaches. Finally, a numerical approach is used to simulate a specific case, where the interface is considered a functionally graded material (FGM).

It is important to highlight that, in the present work, the damage caused by delamination was not considered as a variable, but as an arbitrary value. Therefore, in this specific case, it was assumed that delamination follows a pattern of damage. And, this could be caused by issues in the manufacturing process of the laminate or due to impact loading on the laminated structure in service. Thus, for example, after a NDT (nondestructive test) to inspect the damaged structure, it would be possible to obtain the residual stiffness. In other words, a computer tool could be developed to quantify the influence of delamination in the structure based on data from NDT inspections. 

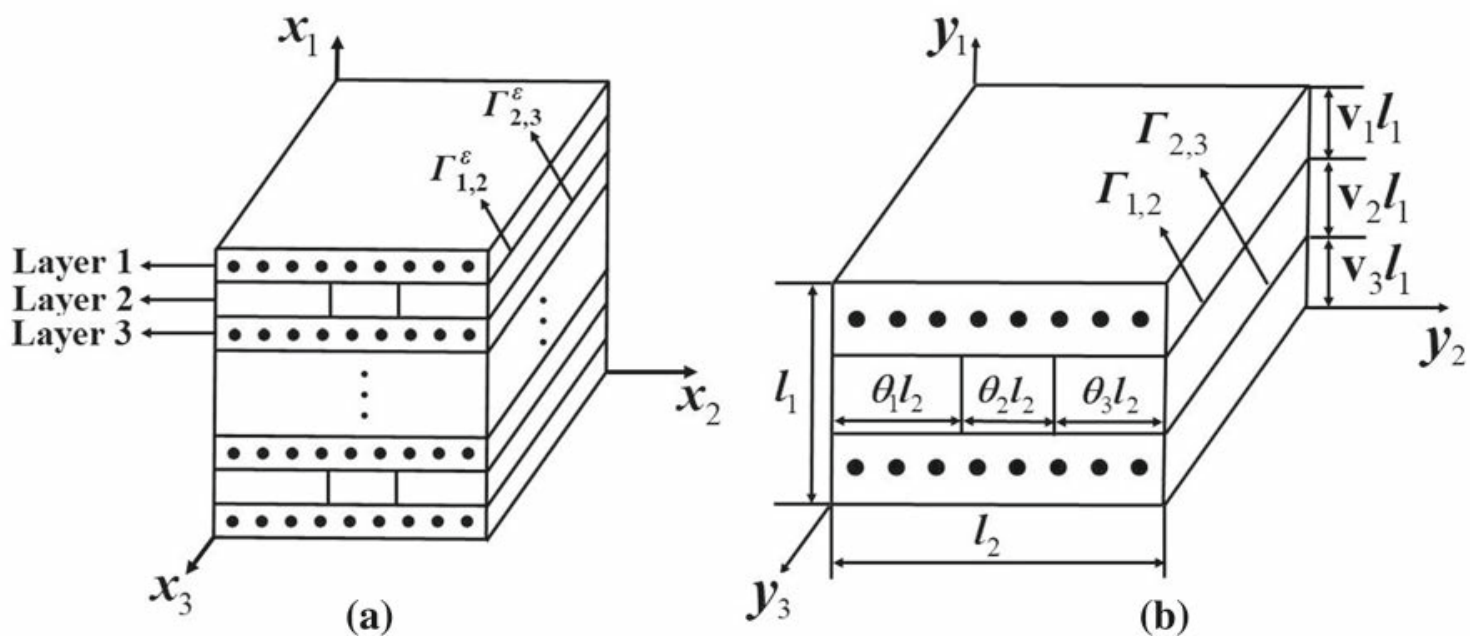

Fig. 1 Analytical model: a laminated composite, b unit cell—-three layers—with delamination in the layer 2

\section{Analytical approach}

\subsection{Geometrical description}

An anisotropic and periodic elastic body is considered. The elastic body occupies a bounded region $\Omega^{\varepsilon}$ in $R^{3}$ space, with Lipschitz boundary $\partial \Omega^{\varepsilon}=\overline{\partial_{1} \Omega^{\varepsilon}} \cup \overline{\partial_{2} \Omega^{\varepsilon}}$, such that $\partial_{1} \Omega^{\varepsilon} \cap \partial_{2} \Omega^{\varepsilon}=\phi$, where $\partial_{1} \Omega^{\varepsilon}$ and $\partial_{2} \Omega^{\varepsilon}$ are boundary portions. Figure 1a shows the 3D laminated composite with composite layers (1 and 3 ) and interface layer represented by layer 2 , where $\Gamma_{\beta, \beta+1}^{\varepsilon}$ is the interface separating the composite layers $\beta$ and $\beta+1(\beta=1,2)$, which depend on the slow variable $\boldsymbol{x}$. $\Gamma_{\beta, \beta+1}$ are the interfaces in the unit cell, which are dependent of the fast variable $\boldsymbol{y}$. The medium is assumed to be stacked in the $x_{1}$ direction (axis normal to the laminate) with all material parameters independent of $x_{2}$ (axis normal to the fibers) and $x_{3}$ (axis aligned to the fibers). Figure $1 \mathrm{~b}$ shows the unit cell, with a localized delamination in the layer 2 (interface), where $\theta_{2} l_{2}$ is the length of delamination and $\theta_{2}$ is the damage degree (the factor of the delamination extension). In addition, it is important to notice that $\theta_{1}+\theta_{2}+\theta_{3}=1$.

\subsection{Statement of the problem}

The problem is formulated in the bounded subset $\Omega^{\varepsilon}$ of $R^{3}$, and $Y=\left\{\boldsymbol{y}=\left(y_{1}, y_{2}, y_{3}\right) \in R^{3}: 0<y_{i}<l_{i}\right.$, $i=1,2,3\}$ denotes the reference cell, with $l_{i}$ being given positive numbers. It is important to notice that $\Omega^{\varepsilon}=\varepsilon Y=\left\{\boldsymbol{x}=\left(x_{1}, x_{2}, x_{3}\right) \in R^{3}: \varepsilon^{-1} x_{i} \in Y, i=1,2,3\right\}$, where $\boldsymbol{y}=\boldsymbol{x} / \varepsilon$.

A general field variable $f_{i}^{\varepsilon}$ is now dependent of both the macro- and microscale, $f_{i}^{\varepsilon}(\boldsymbol{x})=f_{i}(\boldsymbol{x}, \boldsymbol{y})$, and the partial derivatives take the form:

$$
\frac{\partial f_{i}^{\varepsilon}}{\partial x_{j}}=\frac{\partial f_{i}}{\partial x_{j}}+\varepsilon^{-1} \frac{\partial f_{i}}{\partial y_{j}} .
$$

Assuming that the body forces are equal to zero, the elastic equilibrium equation is given by

$$
\frac{\partial \sigma_{i j}^{\varepsilon}}{\partial x_{j}}=0 \text { in } \Omega^{\varepsilon},
$$


and the perfect contact conditions are written as

$$
\begin{aligned}
& {\left[u_{j}^{\varepsilon}\right]_{\Gamma_{\beta, \beta+1}^{\varepsilon}}=\left\{\begin{array}{l}
{\left[u_{j}^{\varepsilon}\right]_{1 \Gamma_{\beta, \beta+1}^{\varepsilon}}=0 \text { in } Y_{1}} \\
{\left[u_{j}^{\varepsilon}\right]_{2 \Gamma_{\beta, \beta+1}^{\varepsilon}}=0 \text { in } Y_{2}} \\
{\left[u_{j}^{\varepsilon}\right]_{3 \Gamma_{\beta, \beta+1}^{\varepsilon}}=0 \text { in } Y_{3}}
\end{array}\right.} \\
& \begin{aligned}
{\left[\sigma_{i j}^{\varepsilon}\right]_{\Gamma_{\beta, \beta+1}^{\varepsilon}} n_{j}=\left\{\begin{array}{l}
{\left[\sigma_{i j}^{\varepsilon}\right]_{1 \Gamma_{\beta, \beta+1}^{\varepsilon}}^{\varepsilon} n_{j}=0 \text { in } Y_{1}} \\
{\left[\sigma_{i j}^{\varepsilon}\right]_{2 \Gamma_{\beta, \beta+1}^{\varepsilon} \Gamma_{j}^{\varepsilon}} n_{j}=0 \text { in } Y_{2}} \\
{\left[\sigma_{i j}^{\varepsilon}\right]_{3 \Gamma_{\beta, \beta+1}^{\varepsilon} n_{j}=0 \text { in } Y_{3}}^{3} n_{j=1}{ }^{2} \Gamma_{\beta, \beta+1}^{\varepsilon}, \beta=1,2}
\end{array}\right.}
\end{aligned} \\
& \sigma_{i j}^{\varepsilon}=C_{i j k l}(\boldsymbol{y}) \frac{\partial u_{k}^{\varepsilon}}{\partial x_{l}}
\end{aligned}
$$

where $\sigma_{i j}^{\varepsilon}$ and $u_{i}^{\varepsilon}$ are the components of the stress tensor and displacement vector, respectively, $[\bullet]_{\Gamma_{\beta, \beta+1}}$ denotes the jump across the interface separating the composite layers $\beta$ and $\beta+1$, i.e., $[\bullet]_{\Gamma_{\beta, \beta+1}}=(\bullet)^{(\beta)}(\boldsymbol{y})-(\bullet)^{(\beta+1)}(\boldsymbol{y})$ for $\boldsymbol{y} \in \Gamma_{\beta, \beta+1}=\bigcup_{r=1}^{3}{ }_{r} \Gamma_{\beta, \beta+1}, \beta=1,2$ (henceforth, the Latin indices take values 1, 2, and 3); $C_{i j k l}=C_{i j k l}^{(1)}$ for $0<y_{1}<v_{1} l_{1}, C_{i j k l}=C_{i j k l}^{(2)}$ for $v_{1} l_{1}<y_{1}<\left(v_{1}+v_{2}\right) l_{1}$ and $C_{i j k l}=C_{i j k l}^{(3)}$ for $\left(v_{1}+v_{2}\right) l_{1}<y_{1}<l_{1}\left(v_{i}\right.$ is the volume fraction of layer $i$, and $l_{1}$ is the length of the unit cell in the $x_{1}$ direction); and $n_{i}$ is the unit vector in the outward normal direction. The symbol $Y_{r}(r=1,2,3)$ (see Definition (22)) represents the $r$-partition of the unit cell $Y$.

\subsection{Two-scale asymptotic homogenization method (AHM)}

Since the 1970s, the mathematical theory of homogenization has developed and is used as an alternative approach to find the effective properties of the equivalent homogenized material [24-27]. It is applicable to all kinds of processes that might occur in periodic media, such as elastic vibrations, heat propagation, diffusion, fluid percolation, electromagnetic oscillations and radiation. From a mathematical point of view, the theory of homogenization is a limit theory which uses the asymptotic expansion and the assumption of periodicity to substitute the differential equations with rapidly oscillating coefficients, with differential equations whose coefficients are constant or slowly varying in such a way that the solutions are close to the initial equations [26].

Using the double-scale asymptotic expansion, the displacements $\left(u_{i}^{\varepsilon}(\boldsymbol{x})\right)$ are expressed as [25-27]:

$$
u_{i}^{\varepsilon}(\boldsymbol{x})=u_{i}^{(0)}(\boldsymbol{x})+\varepsilon \cdot u_{i}^{(1)}(\boldsymbol{x}, \boldsymbol{y})+\varepsilon^{2} \cdot u_{i}^{(2)}(\boldsymbol{x}, \boldsymbol{y})+\cdots .
$$

Substituting (5) into (4), it is possible to obtain

$$
\sigma_{i j}^{\varepsilon}(\boldsymbol{x})=\sigma_{i j}^{(0)}(\boldsymbol{x}, \boldsymbol{y})+\varepsilon \cdot \sigma_{i j}^{(1)}(\boldsymbol{x}, \boldsymbol{y})+\varepsilon^{2} \cdot \sigma_{i j}^{(2)}(\boldsymbol{x}, \boldsymbol{y})+\cdots
$$

where

$$
\sigma_{i j}^{(m)}(\boldsymbol{x}, \boldsymbol{y})=C_{i j k l}(\boldsymbol{y}) \frac{\partial u_{k}^{(m)}}{\partial x_{l}}+C_{i j k l}(\boldsymbol{y}) \frac{\partial u_{k}^{(m+1)}}{\partial y_{l}}, \quad m=0,1,2, \ldots
$$

Substituting expressions (5) and (6) into (2) and rearranging the terms of equal exponent $\varepsilon$, it are obtained for $\varepsilon^{-1}$ and $\varepsilon^{0}$ the following equations:

$$
\frac{\partial \sigma_{i j}^{(0)}}{\partial y_{j}}=0
$$




$$
\frac{\partial \sigma_{i j}^{(0)}}{\partial x_{j}}+\frac{\partial \sigma_{i j}^{(1)}}{\partial y_{j}}=0 .
$$

Using Eq. (7) for $m=0$ and Eq. (8), they yield

$$
\frac{\partial}{\partial y_{j}}\left(C_{i j k l} \frac{\partial u_{k}^{(1)}}{\partial y_{l}}\right)=-\frac{\partial C_{i j k l}}{\partial y_{j}} \frac{\partial u_{k}^{(0)}}{\partial x_{l}}
$$

where the solution is represented in the form

$$
u_{i}^{(1)}(\boldsymbol{x}, \boldsymbol{y})=\chi_{i}^{j k}(\boldsymbol{y}) \frac{\partial u_{j}^{(0)}(\boldsymbol{x})}{\partial x_{k}}
$$

and thus, one obtains the following local problems for determining the $l_{1}$-periodic auxiliary functions $\chi_{i}^{j k}(y)$;

$$
\frac{\partial}{\partial y_{j}}\left(C_{i j k l} \frac{\partial \chi_{k}^{p q}}{\partial y_{l}}+C_{i j p q}\right)=0 \text { in } Y,
$$

with interface conditions

$$
\left[\chi_{k}^{p q}\right]_{\Gamma_{\beta, \beta+1}}=0,\left[C_{i j k l} \frac{\partial \chi_{k}^{p q}}{\partial y_{l}}+C_{i j p q}\right]_{\Gamma_{\beta, \beta+1}} n_{j}=0 \text { on } \Gamma_{\beta, \beta+1}=\bigcup_{r=1}^{3} r \Gamma_{\beta, \beta+1}
$$

where the auxiliary functions $\chi_{k}^{p q}(y)$ are piecewise linear functions in each unit cell portion $Y_{r}$, which are defined as:

$$
\chi_{k}^{p q}= \begin{cases}1 \chi_{k}^{p q} & \text { in } Y_{1} \\ 2 \chi_{k}^{p q} & \text { in } Y_{2} \\ 3 \chi_{k}^{p q} & \text { in } Y_{3}\end{cases}
$$

with the $r$-interface-partition local functions ${ }_{r} \chi_{k}^{p q}(r=1,2,3)$.

The existence of the local functions $\chi_{k}^{p q}$ is guaranteed up to an additive constant (cf. Lemma 1 in [27]). To ensure uniqueness, it is taken $\chi_{k}^{p q}$ so that $\left\langle\chi_{k}^{p q}\right\rangle_{Y}=0$. The cell average operator $\langle\bullet\rangle_{Y}$ is defined as $\langle\bullet\rangle_{Y}=\frac{1}{|Y|} \int_{Y} \bullet \mathrm{d} Y$, where $|Y|$ is the unit cell volume.

Note that the local problems (12)-(13) can also be obtained from the following equilibrium states:

$$
\int_{Y} C_{i j p q} \frac{\partial \chi_{p}^{k l}}{\partial y_{q}} \frac{\partial v_{i}}{\partial y_{j}} \mathrm{~d} Y=-\int_{Y} C_{i j k l} \frac{\partial v_{i}}{\partial y_{j}} \mathrm{~d} Y
$$

where $\boldsymbol{v}$ is a virtual displacement field.

From Eq. (7) for $m=0$ and considering (11), it is obtained

$$
\sigma_{i j}^{(0)}=\left(C_{i j p q}+C_{i j k l} \frac{\partial \chi_{k}^{p q}}{\partial y_{l}}\right) \frac{\partial u_{p}^{(0)}}{\partial x_{q}} .
$$

The macroscopic equilibrium equation is obtained by averaging Eq. (9), and therefore,

$$
\frac{\partial\left\langle\sigma_{i j}^{(0)}\right\rangle_{Y}}{\partial x_{j}}=0
$$

where

$$
\left\langle\sigma_{i j}^{(0)}\right\rangle_{Y}=\frac{1}{|Y|} \int_{V} \sigma_{i j}^{(0)} \mathrm{d} Y=C_{i j k l}^{*} \frac{\partial u_{k}^{(0)}}{\partial x_{l}},
$$


with

$$
C_{i j k l}^{*}=\left\langle C_{i j k l}+C_{i j h s} \frac{\partial \chi_{h}^{k l}}{\partial y_{s}}\right\rangle_{Y} .
$$

Proposition Expression (19) for the effective moduli $C_{i j k l}^{*}$ can be written as $C_{i j k l}^{*}=\sum_{r=1}^{3} \theta_{r}\left\langle C_{i j k l}+C_{i j h 1} \frac{\partial_{r} \chi_{h}^{k l}}{\partial y_{1}}\right\rangle_{Y_{r}}$ where $\theta_{r}$ denotes the degree of imperfection of the r-partition.

Proof Considering (19) and keeping in mind (16),

$$
\begin{aligned}
& C_{i j k l}^{*}=\left\langle C_{i j k l}+C_{i j h s} \frac{\partial \chi_{h}^{k l}}{\partial y_{s}}\right\rangle_{Y}=\frac{1}{l_{1} l_{2}} \int_{0}^{l_{1}} \int_{0}^{l_{2}}\left(C_{i j k l}+C_{i j h s} \frac{\partial \chi_{h}^{k l}}{\partial y_{s}}\right) \mathrm{d} y_{1} \mathrm{~d} y_{2}, \\
& C_{i j k l}^{*}=\frac{1}{l_{1} l_{2}} \sum_{r=1}^{3} \int_{0}^{l_{1}} \int_{0}^{\theta_{r} l_{2}}\left(C_{i j k l}+C_{i j h s} \frac{\partial_{r} \chi_{h}^{k l}}{\partial y_{s}}\right) \mathrm{d} y_{1} \mathrm{~d} y_{2}=\sum_{r=1}^{3} \frac{\theta_{r}}{l_{1}} \int_{0}^{l_{1}}\left(C_{i j k l}+C_{i j h s} \frac{\partial_{r} \chi_{h}^{k l}}{\partial y_{s}}\right) \mathrm{d} y_{1},
\end{aligned}
$$

finally, it is possible to obtain

$$
C_{i j k l}^{*}=\sum_{r=1}^{3} \frac{\theta_{r}}{l_{1}} \int_{0}^{l_{1}}\left(C_{i j k l}+C_{i j h s} \frac{\partial_{r} \chi_{h}^{k l}}{\partial y_{s}}\right) \mathrm{d} y_{1}=\sum_{r=1}^{3} \theta_{r}\left\langle C_{i j k l}+C_{i j h 1} \frac{\partial_{r} \chi_{h}^{k l}}{\partial y_{1}}\right\rangle_{Y_{r}} .
$$

From (12), (13), one obtains the following local problems for each $r$-interface partition:

$$
\frac{\partial}{\partial y_{j}}\left(C_{i j k l} \frac{\partial_{r} \chi_{k}^{p q}}{\partial y_{l}}+C_{i j p q}\right)=0 \text { in } Y_{r}
$$

with interface conditions

$$
\left[{ }_{r} \chi_{k}^{p q}\right]_{\Gamma_{\beta, \beta+1}}=0,\left[C_{i j k l} \frac{\partial_{r} \chi_{k}^{p q}}{\partial y_{l}}+C_{i j p q}\right]_{\Gamma_{\beta, \beta+1}} n_{j}=0 \text { on } \Gamma_{\beta, \beta+1}, \beta=1,2
$$

where

$$
Y_{r}=\left\{\boldsymbol{y}=\left(y_{1}, y_{2}, y_{3}\right) \in \mathbb{R}^{3}: 0<y_{i}<l_{i}, i=1,3 \text { and } l_{2} \sum_{t=0}^{r-1} \theta_{t}<y_{2}<l_{2} \sum_{t=1}^{r} \theta_{t}, \theta_{0}=0\right\},
$$

and $\Gamma_{r}$ is the interface of the unit cell portion $Y_{r}$.

The nonzero local functions $r \chi_{i}^{j k}$ are calculated for orthotropic constituents, and using (19), the effective moduli can be written as

$$
\begin{aligned}
& C_{1111}^{*}=\sum_{r=1}^{3} \theta_{r}\left\langle\frac{1}{C_{1111}}\right\rangle_{Y_{r}}^{-1}, \\
& C_{2222}^{*}=\sum_{r=1}^{3} \theta_{r}\left(\left\langle C_{2222}\right\rangle_{Y_{r}}+\left\langle\frac{1}{C_{1111}}\right\rangle_{Y_{r}}^{-1}\left\langle\frac{C_{1122}}{C_{1111}}\right\rangle_{Y_{r}}^{2}-\left\langle\frac{\left(C_{1122}\right)^{2}}{C_{1111}}\right\rangle_{Y_{r}}\right), \\
& C_{3333}^{*}=\sum_{r=1}^{3} \theta_{r}\left(\left\langle C_{3333}\right\rangle_{Y_{r}}+\left\langle\frac{1}{C_{1111}}\right\rangle_{Y_{r}}^{-1}\left\langle\frac{C_{1133}}{C_{1111}}\right\rangle_{Y_{r}}^{2}-\left\langle\frac{\left(C_{1133}\right)^{2}}{C_{1111}}\right\rangle_{Y_{r}}\right), \\
& C_{1122}^{*}=\sum_{r=1}^{3} \theta_{r}\left\langle\frac{1}{C_{1111}}\right\rangle_{Y_{r}}^{-1}\left\langle\frac{C_{1122}}{C_{1111}}\right\rangle_{Y_{r}},
\end{aligned}
$$




$$
\begin{aligned}
& C_{1133}^{*}=\sum_{r=1}^{3} \theta_{r}\left\langle\frac{1}{C_{1111}}\right\rangle_{Y_{r}}^{-1}\left\langle\frac{C_{1133}}{C_{1111}}\right\rangle_{Y_{r}}, \\
& C_{2233}^{*}=\sum_{r=1}^{3} \theta_{r}\left(\left\langle C_{2233}\right\rangle_{Y_{r}}+\left\langle\frac{1}{C_{1111}}\right\rangle_{Y_{r}}^{-1}\left\langle\frac{C_{1122}}{C_{1111}}\right\rangle_{Y_{r}}\left\langle\frac{C_{1133}}{C_{1111}}\right\rangle_{Y_{r}}-\left\langle\frac{C_{1122} C_{1133}}{C_{1111}}\right\rangle_{Y_{r}}\right), \\
& C_{1212}^{*}=\sum_{r=1}^{3} \theta_{r}\left\langle\frac{1}{C_{1212}}\right\rangle_{Y_{r}}^{-1}, C_{1313}^{*}=\sum_{r=1}^{3} \theta_{r}\left\langle\frac{1}{C_{1313}}\right\rangle_{Y_{r}}^{-1}, C_{2323}^{*}=\frac{1}{2}\left[C_{2222}^{*}-C_{2233}^{*}\right] .
\end{aligned}
$$

\section{Numerical approach}

\subsection{Finite element method: formulation}

In this Section, Eqs. (15) and (19) are discretized by finite elements and solved by the imposition of a periodic displacement applied directly in the global arrays of the problem [28,29]. Thus, the equilibrium problem, stated in Eq. (15), can be written in a vectorial form as:

$$
\int_{Y}\left(\frac{\partial \boldsymbol{v}}{\partial \boldsymbol{y}}\right)^{T} \boldsymbol{C} \frac{\partial \chi}{\partial \boldsymbol{y}} \mathrm{d} Y=-\int_{Y}\left(\frac{\partial \boldsymbol{v}}{\partial \boldsymbol{y}}\right)^{T} \boldsymbol{C} \mathrm{d} Y .
$$

If the representative volume element is divided into a finite number of finite elements, the virtual displacements $\boldsymbol{v}$ and the characteristic solution $\chi$ can be written as [30]:

$$
\boldsymbol{v}=\boldsymbol{N}_{\alpha}^{g} \hat{\boldsymbol{v}}_{\alpha}=\boldsymbol{N}^{g} \hat{\boldsymbol{v}}, \alpha=1,2, \ldots, N_{E L},
$$

and

$$
\chi=N_{\alpha}^{g} \hat{\chi}_{\alpha}=N^{g} \hat{\chi}, \alpha=1,2, \ldots, N_{E L}
$$

where $N_{\alpha}^{g}$ is the global shape function associated with the $\alpha$ th node of the mesh and element type, $N_{E L}$ is the total number of elements used in the RVE discretization, and $\hat{v}$ and $\hat{\chi}$ are, respectively, the nodal virtual displacement vector and the nodal characteristic displacement vector. By using the differential linear operator, which is given by

$$
\boldsymbol{L}=\left[\begin{array}{ccc}
\frac{\partial}{\partial y_{1}} & 0 & 0 \\
0 & \frac{\partial}{\partial y_{2}} & 0 \\
0 & 0 & \frac{\partial}{\partial y_{3}} \\
\frac{\partial}{\partial y_{3}} & 0 & \frac{\partial}{\partial y_{1}} \\
0 & \frac{\partial}{\partial y_{3}} & \frac{\partial}{\partial y_{2}} \\
\frac{\partial}{\partial y_{2}} & \frac{\partial}{\partial y_{1}} & 0
\end{array}\right],
$$

the global strain-displacement matrix can be written as:

$$
\boldsymbol{B}=\boldsymbol{L} \boldsymbol{N}^{g} .
$$

Therefore, Eq. (28) is used to obtain the derivatives of the virtual displacement field and the derivatives of the characteristic displacements as:

$$
\frac{\partial v}{\partial y}=\boldsymbol{L} \boldsymbol{v}=\boldsymbol{L} \boldsymbol{N}^{g} \hat{\boldsymbol{v}}=\boldsymbol{B} \hat{\boldsymbol{v}}
$$


and

$$
\frac{\partial \chi}{\partial \boldsymbol{y}}=\boldsymbol{L} \chi=L \boldsymbol{N}^{g} \hat{\chi}=\boldsymbol{B} \hat{\chi} .
$$

Equations (29) and (30) are now used in the equilibrium Eq. (24), resulting in

$$
\int_{Y}(\boldsymbol{B} \hat{\boldsymbol{v}})^{T} \boldsymbol{C B} \hat{\chi} \mathrm{d} Y=-\int_{Y}(\boldsymbol{B} \hat{\boldsymbol{v}})^{T} \boldsymbol{C} \mathrm{d} Y .
$$

Since the virtual displacements are arbitrary, they can be taken from the integral, leading to

$$
\hat{\boldsymbol{v}}^{T} \int_{Y} \boldsymbol{B}^{T} \boldsymbol{C B} \hat{\chi} \mathrm{d} Y=-\hat{\boldsymbol{v}}^{T} \int_{Y} \boldsymbol{B}^{T} \boldsymbol{C} \mathrm{d} Y
$$

and be chosen in a such way that

$$
\int_{Y} \boldsymbol{B}^{T} \boldsymbol{C B} \hat{\chi} \mathrm{d} Y=-\int_{Y} \boldsymbol{B}^{T} \boldsymbol{C} \mathrm{d} Y .
$$

Equation (33) resembles a standard finite element equation, and it can be written as:

$$
K \chi=-f
$$

where $\boldsymbol{K}$ is the RVE stiffness matrix, $\chi$ is the characteristic periodic displacement field and $\boldsymbol{f}$ is the homogenization load vector. This equation represents the global equilibrium of the RVE when submitted to the homogenized load.

For a single element of the mesh, the local stiffness matrix is given by:

$$
\boldsymbol{K}^{e}=\int_{Y^{e}} \boldsymbol{B}^{e T} \boldsymbol{C}^{e} \boldsymbol{B}^{e} \mathrm{~d} Y .
$$

And, by using a quadrature scheme to evaluate the integral, Eq. (35) can be written as:

$$
\boldsymbol{K}^{e}=\sum_{m=1}^{p}\left(\mathbf{B}_{m}^{e T} \boldsymbol{C}^{e} \mathbf{B}_{m}^{e}\right) W_{m} J_{m}
$$

where $p$ is the number of quadrature points used in the integration, $W_{m}$ and $J_{m}$ are, respectively, the quadrature weight and the determinant of the Jacobian matrix, which is associated with the $m$ th quadrature point, and the super-index $e$ indicates the local arrays. The local homogenized load vector, for a single element of the mesh, is given by:

$$
\boldsymbol{f}^{e}=\int_{Y} \boldsymbol{B}^{e T} \boldsymbol{C}^{e} \mathrm{~d} Y .
$$

And, by using a quadrature scheme, it is possible to have:

$$
\boldsymbol{f}^{e}=\sum_{m=1}^{p}\left(\mathbf{B}_{m}^{e T} \boldsymbol{C}^{e}\right) W_{m} J_{m} .
$$

The global arrays are assembled in the standard way as:

$$
\boldsymbol{K}=\bigcup_{e=1}^{N_{E L}} \boldsymbol{K}^{e}
$$


and

$$
\boldsymbol{f}=\bigcup_{e=1}^{N_{E L}} \boldsymbol{f}^{e}
$$

Periodicity constraints are applied directly in the global arrays, Eqs. (39) and (40), in order to obtain the periodic displacement field $\chi$.

A similar procedure is used in order to discretize the integral Eq. (18), and it can be written as:

$$
\boldsymbol{C}^{*}=\frac{1}{|Y|} \int_{Y}(\boldsymbol{C}+\boldsymbol{C B} \hat{\chi}) \mathrm{d} Y .
$$

The integral Eq. (41) can be substituted by a sum through all elements of the mesh and solved by a quadrature scheme, as well:

$$
C_{i j k l}^{*}=\sum_{e=1}^{N} \sum_{m=1}^{p}\left(C_{i j k l}^{e}+C_{i j r s}^{e} B_{r}^{(e)(m)} \chi_{s}^{k l(e)}\right) W_{m} J_{m} .
$$

Thus, the equilibrium equation (34) is solved alongside Eqs. (39) and (40) for six load cases (tractions in the three directions $-y_{1}, y_{2}$, and $y_{3}$; shear on the three planes- $y_{1} y_{2}, y_{1} y_{3}$, and $\left.y_{2} y_{3}\right)$ and used in Eq. (42) in order to obtain the homogenized fourth-order elasticity tensor of the medium.

\subsection{Finite Element Method: Implementation and RVE}

A three-dimensional in-house finite element software, written in Julia language [31], was developed in order to evaluate numerically the effective mechanical coefficients of the laminated composite with and without delamination.

To mesh the domain, it was chosen the trilinear isoparametric hexahedral element with incompatible modes [32]. This element has the same shape functions as the regular eight-node trilinear isoparametric element, with three additional quadratic shape functions, one for each natural coordinate. The advantage is improving the convergence rate without compromising the computational effort since both local stiffness matrices have the same size. Figure 2 shows the RVE finite element model used to carry out the numerical simulations. The white and dark gray parts play the role of orthotropic plies, and the light gray material represents the epoxy layer (interface between the layers). The delamination is modeled by a penalization in the mechanical fourth-order elasticity tensor of the interface, such that the influence of the stiffness of the penalized elements becomes negligible, i.e., with stiffness close to zero for selected elements. Thus, as more finite elements are penalized, the delamination has more extension. For the sake of simplicity in mesh generation, the elements in each individual layer are regular.

A mesh convergence analysis was performed for both intact and completely damaged RVE with a thickness of the intermediate layer of $0.05\left(t^{(2)}=0.05\right)$. The difference found in all components of the fourth-order elasticity tensor, by using finite element meshes of $10 \times 10 \times 10,20 \times 20 \times 20,30 \times 30 \times 30$, and $50 \times 50 \times 50$ elements, was in the order of $10^{-6}$. This result shows that there is almost no influence of the mesh refinement on the numerical results. In addition, as the computational effort is considerably low for the coarse meshes, the finite element mesh is thus chosen to guarantee an aspect ratio lower than 3 (three) in the middle layer in order to avoid numerical issues.

\section{Results}

In order to evaluate the effect of delamination on the effective properties by using numerical (FEM) and analytical (AHM) methods, some investigations are presented. Thus, four different stacking sequences $\left([0]_{n}\right.$, $\left.[0 / 90]_{n},[30 /-30]_{n},[45 /-45]_{n}\right)$ are considered. The material properties used in the calculations are given in Tables 1 and 2, which are given by the literature [34,35]. 


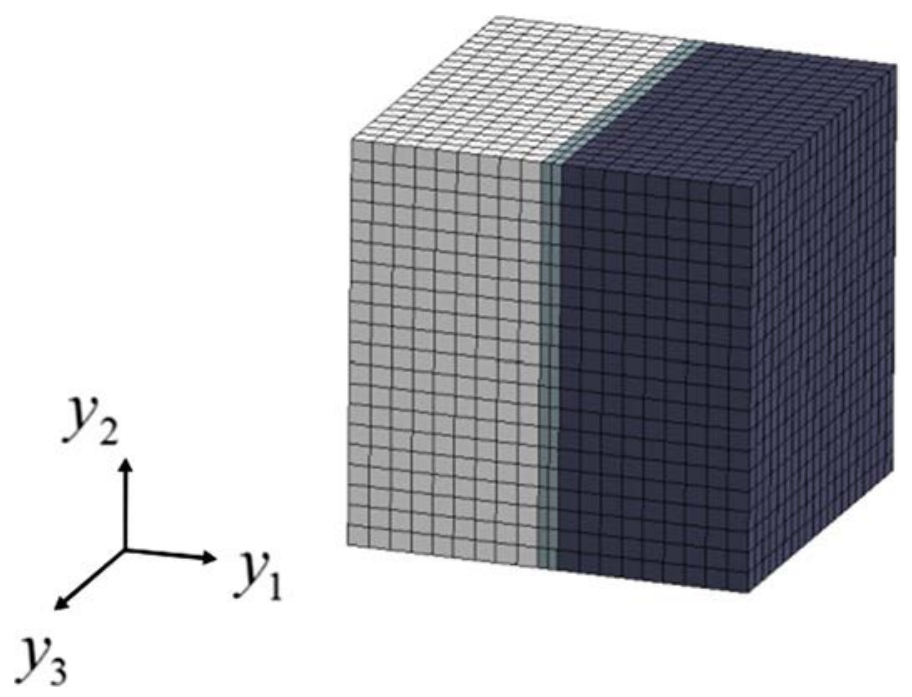

Fig. 2 Finite element model: mesh of the RVE with three layers

Table 1 Mechanical properties of the orthotropic plies (unidirectional carbon fiber embeded in an epoxy matrix) [34]

\begin{tabular}{lc}
\hline$E_{T}(\mathrm{GPa})$ & 10.0 \\
$E_{L}(\mathrm{GPa})$ & 127.0 \\
$\nu_{L T}$ & 0.306 \\
$\nu_{T L}(\mathrm{GPa})$ & 0.34 \\
$G_{L T}(\mathrm{GPa})$ & 3.05 \\
$G_{T L}$ & 5.4 \\
\hline
\end{tabular}

$L$ longitudinal, $T$ transversal

Table 2 Interface properties (epoxy) [35]

\begin{tabular}{ll}
\hline$E(\mathrm{GPa})$ & 5.033 \\
$\nu$ & 0.4 \\
\hline
\end{tabular}

\subsection{Limit cases analysis}

In Figs. 3, 4, 5, and 6, the effective coefficients for the investigated laminates considered without delamination $\left(\theta_{2}=0\right)$ and complete delamination $\left(\theta_{2}=1\right)$ and for different values of the interface thickness $t^{(2)}=v_{2} l_{1}$ are shown. Based on the values of the effective coefficients, it can be seen that for both methods (FEM and AHM), the results show good agreement, because numerical analyses use an asymptotic homogenization approach as the analytical ones. This can be confirmed by comparing Eqs. (19) and (41).

Almost all effective coefficients, except the effective coefficients $C_{12}^{*}, C_{13}^{*}$, and $C_{23}^{*}$, decrease as the layer 2 (interface) thickness increases for the case of a perfect interface $\left(\theta_{2}=0\right)$. Since the Young's and shear moduli of the orthotropic plies (layers 1 and 3) are considerably higher than the elastic properties of the interface (layer 2 ), as the thickness of the interface increases, a smaller volume fraction of the orthotropic layers contributes to the laminate behavior. Thus, the effective coefficients in the main diagonal of the elastic tensor decrease, because these coefficients are mainly related to the Young's and shear moduli of the constituents. On the other hand, the coupled behavior of the interface is more pronounced than the coupled behavior of the orthotropic plies, since its Poisson's coefficient is higher. Thus, as the interface thickness increases, more volume fraction of layer 2 contributes to the coupled effect in the laminated composite, which is more pronounced, increasing the values of coefficients $C_{12}^{*}, C_{13}^{*}$, and $C_{23}^{*}$.

The interface properties are negligible for the case of complete delamination $\left(\theta_{2}=1\right)$. Thus, the analyses are performed only considering the influence of the orthotropic plies. First, some coefficients become close to zero $\left(C_{11}^{*}, C_{12}^{*}, C_{13}^{*}, C_{55}^{*}\right.$, and $\left.C_{66}^{*}\right)$ due to the absence of interface between layers. Second, all effective 

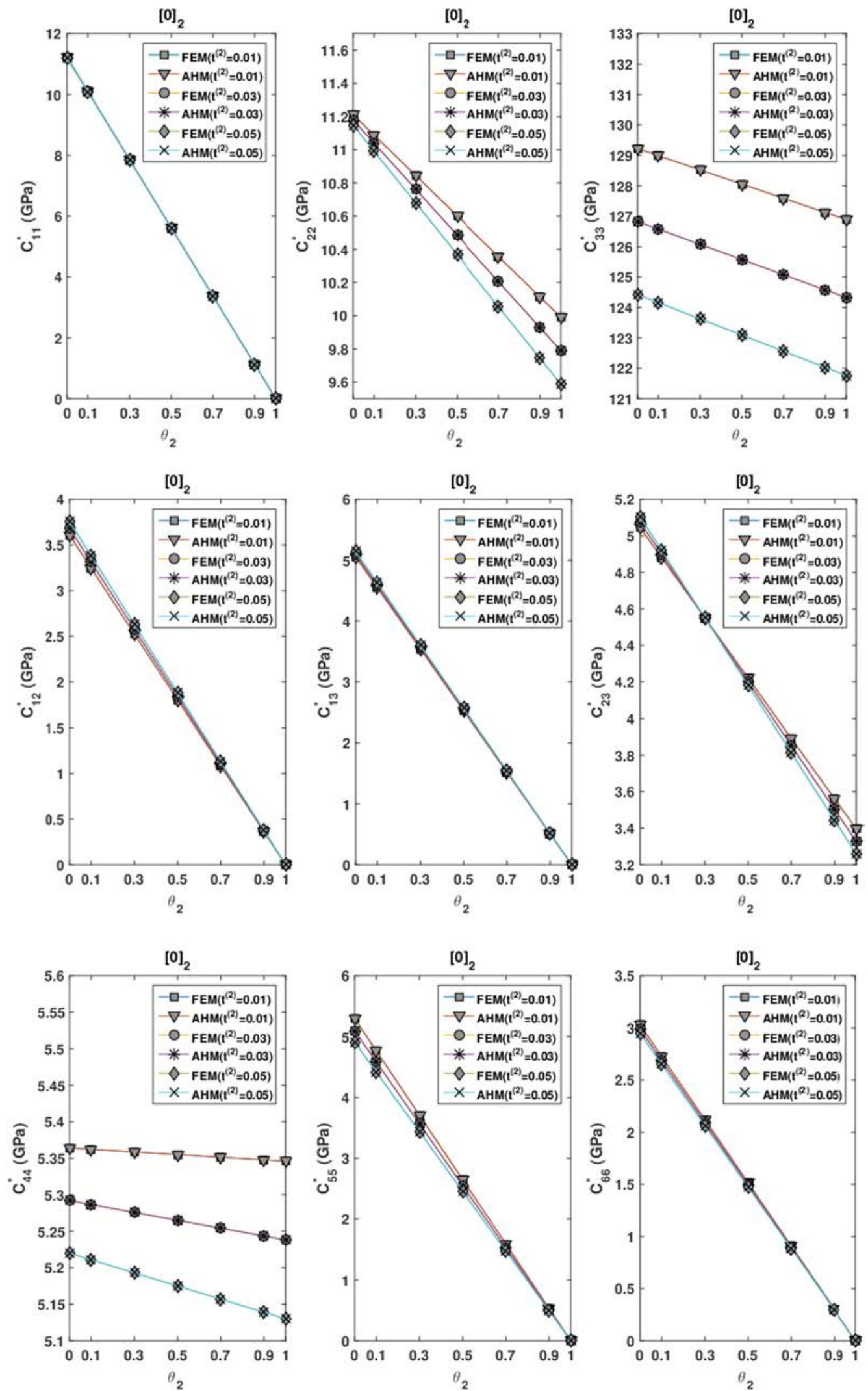

Fig. 3 Effective coefficients for the stacking sequence $[0]_{n}$ as functions of the damage degree $\theta_{2}$ for different values of the interface thickness $t^{(2)}$ 

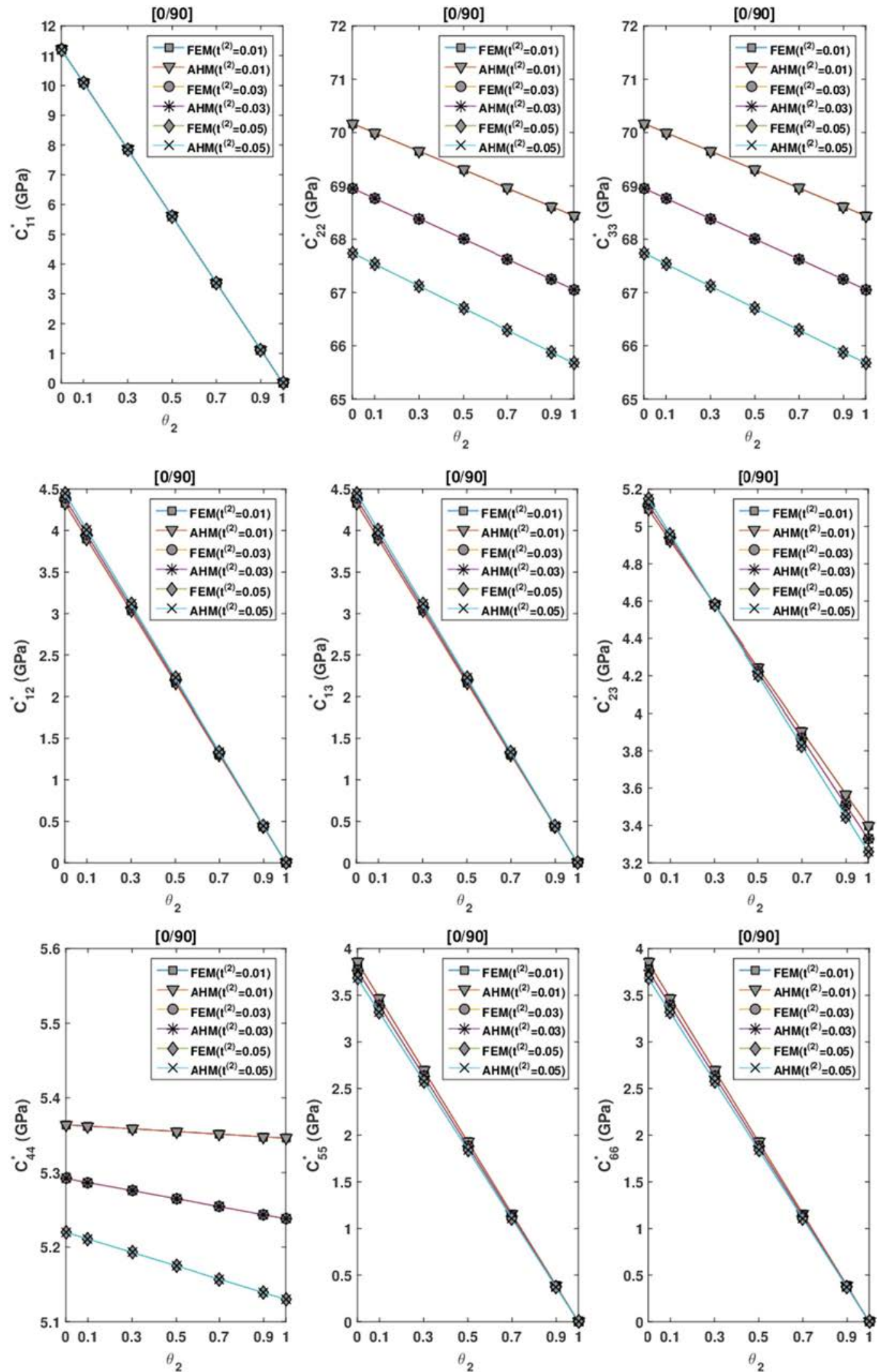

Fig. 4 Effective coefficients for the stacking sequence $[0 / 90]_{n}$ as functions of the damage degree $\theta_{2}$ for different values of the interface thickness $t^{(2)}$ 

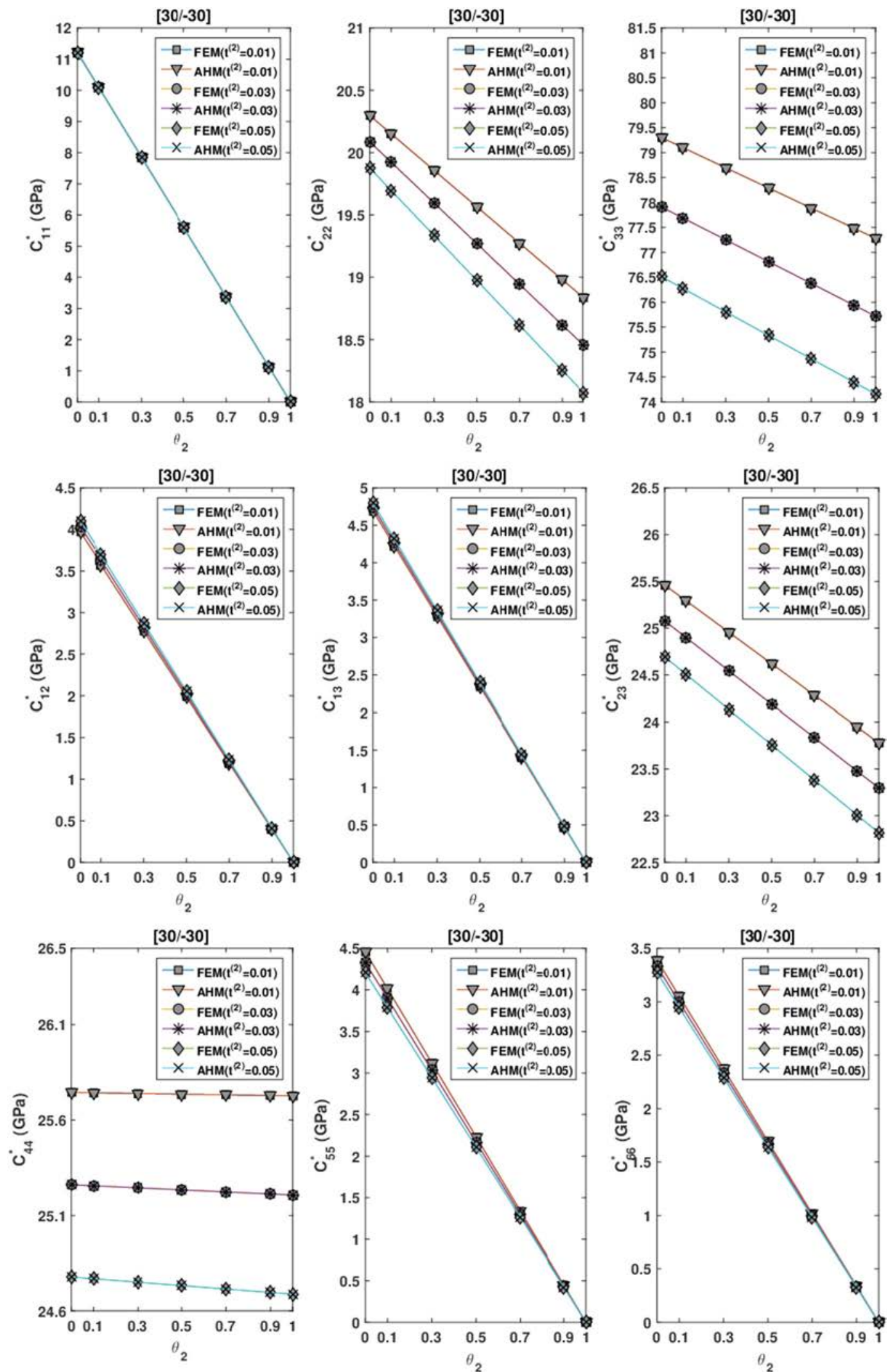

Fig. 5 Effective coefficients for the stacking sequence $[30 /-30]_{n}$ as functions of the damage degree $\theta_{2}$ for different values of the interface thickness $t^{(2)}$ 

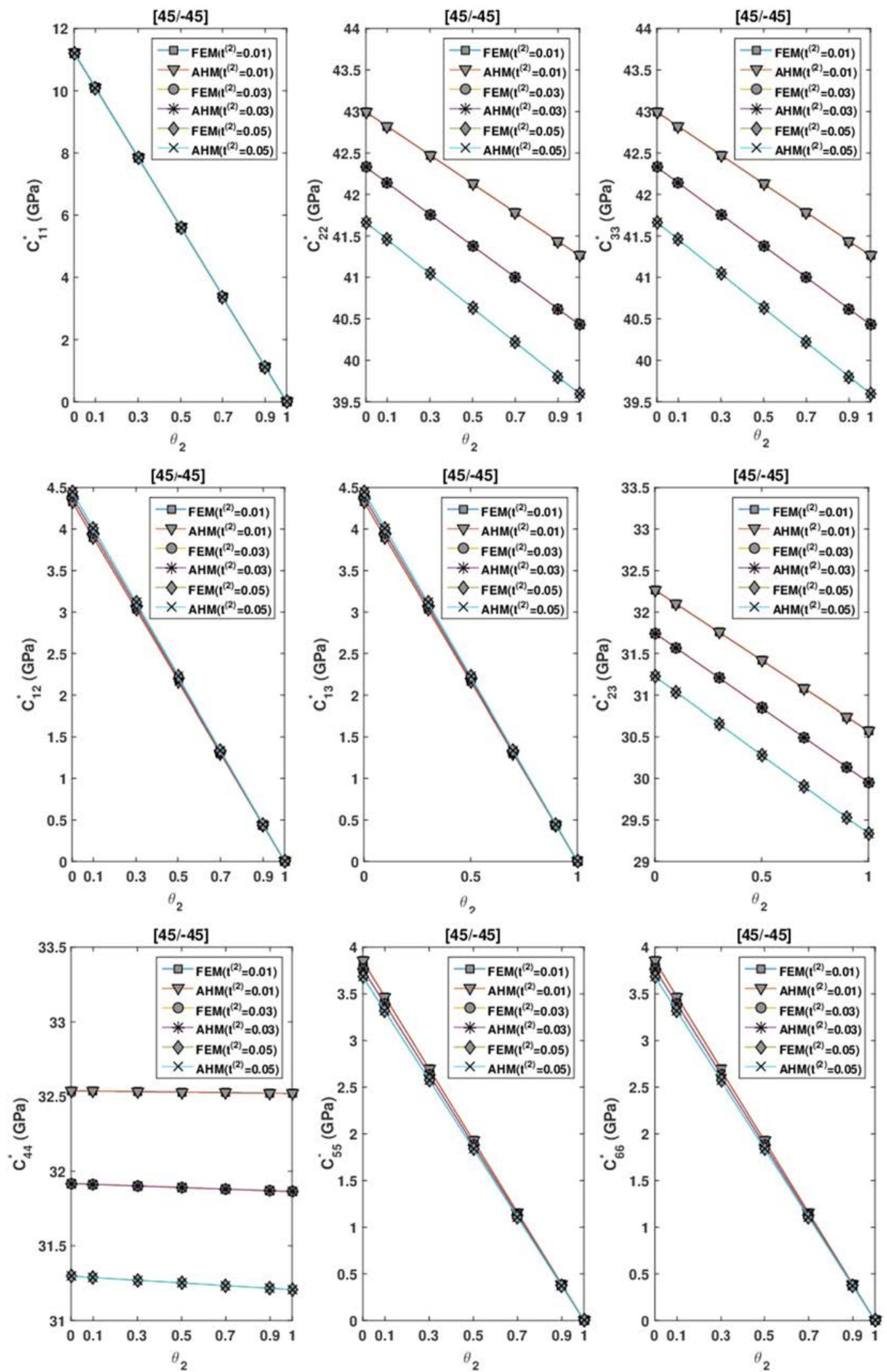

Fig. 6 Effective coefficients for the stacking sequence $[45 /-45]_{n}$ as functions of the damage degree $\theta_{2}$ for different values of the interface thickness $t^{(2)}$ 
Table 3 The effective coefficients for the stacking sequences [0/90] $n$ with interface thickness $t^{(2)}=0.05 \mathrm{~mm}$

\begin{tabular}{llllllllll}
\hline Power law & $C_{11}^{*}$ & $C_{22}^{*}$ & $C_{33}^{*}$ & $C_{12}^{*}$ & $C_{13}^{*}$ & $C_{23}^{*}$ & $C_{44}^{*}$ & $C_{55}^{*}$ & $C_{66}^{*}$ \\
\hline Linear & 10.213880 & 67.415870 & 67.429210 & 4.046107 & 4.052850 & 4.921269 & 5.173188 & 3.235262 & 3.247179 \\
Quadratic & 8.877800 & 67.154235 & 67.168137 & 3.514536 & 3.521776 & 4.689919 & 5.158191 & 2.808086 & 2.850174 \\
Cubic & 7.693238 & 66.943035 & 66.956411 & 3.044280 & 3.051341 & 4.493357 & 5.150776 & 2.471367 & 2.533422 \\
\hline
\end{tabular}

coefficients decrease as the layer 2 thickness increases, due to the fact that the volume fraction of the orthotropic plies decreases, and this reduces all effective coefficients of the laminate.

\subsection{Delamination effects and influence of the stacking sequence}

Figures $3,4,5$, and 6 show the delamination process simulation for different values of the interface thickness $t^{(2)}$. The values of the effective coefficients for the main diagonal of the mechanical tensor are similar to the limit cases, decreasing as the thickness of layer 2 decreases, for intermediary values of damage extension. The coupled components for the perfect adhesion (without delamination) and for the complete separation of layers present opposite behavior.

In addition, it can be seen that, as the delamination degree increases, all coefficients decrease and the most affected coefficients are those dependent on the lamination direction $\left(y_{1}\right)$. The influence of the stacking sequence of the laminated composites is relevant. For the stacking sequences $[0]_{n},[0 / 90]_{n}$ and $[45 /-45]_{n}$, the global mechanical properties behave as a purely orthotropic material (transversally isotropic). The case $[30 /-30]_{n}$ exhibits an anisotropic behavior.

4.3 Effective coefficients considering the interface between two layers as functionally graded material (FGM)

After evaluating the potentialities and limitations of the numerical approach, there are calculated the effective properties for laminated composites, considering the properties of the interface between two layers as functionally graded material (FGM). Thus, the variation of the interface properties is according to the law $\left(y_{2} / l_{2}\right)^{p} C_{i j k l}^{(2)}$ with $p=1,2,3$. Solving this problem, using the analytical technique of the asymptotic homogenization method (AHM), requires a great effort to obtain not only the solution of the local problems, but also the analytical expressions of the effective coefficients. This problem can be faced by the finite element method (FEM), which can be applied with accuracy to determine the effective coefficients. The effective stiffness for the stacking sequences $[0 / 90]_{n}$ with interface thickness $t^{(2)}=0.05 \mathrm{~mm}$ is given in Table 3 . It is observed that if the value of the exponent $p$ increases, then the effective coefficients decrease. In other words, the degree of variation of the material properties in the interface of the layers affects the value of the effective coefficients.

Finally, it is worth to highlight that for some specific delamination problems the calculation of the effective coefficients via asymptotic homogenization method (AHM) does not show physical consistency, because the present approach is not able to capture the effect of the location of the delamination. In fact, the coefficients for different damage degree (delamination extension) are calculated by AHM, averaging the results of limit cases: the complete separation of the interface and perfect bonding. In other words, AHM (as it is written in the present paper) predicts the same effective coefficients for delaminated composites having the same extension of delamination, but with different damage locations. This problem can be faced by the finite element method (FEM), which can be applied with accuracy to determine the effective coefficients for delaminated composites, considering not only the extension of damage but also its location.

\section{Conclusions}

This work is focused on the analysis of two approaches (numerical and analytical) in macroscale level to determine the effective properties of laminated composites considering localized delaminations in the interface between two plies. It is shown that the results obtained by both methods, asymptotic homogenization method (AHM) and finite element method (FEM), have a good agreement. In addition, it is possible to conclude that the effective coefficient values decrease as the damage extension increases due to the fact that the delamination 
area increases. Besides, for all effective coefficients, except the effective coefficients $C_{12}^{*}, C_{13}^{*}$, and $C_{23}^{*}$, in the case without delamination, the coefficients decrease as the layer 2 (adhesive region) thickness increases, and almost all coefficients decrease for complete separation of the interface. In general, the effective elastic moduli tensor for the laminates $[0]_{n},[0 / 90]_{n}$ and $[45 /-45]_{n}$ is coherent with the mechanical behavior of a laminated composite shown by the literature.

For some specific delamination problems, the calculation of the effective coefficients via asymptotic homogenization method (AHM) does not show physical consistency, because the present approach is not able to capture the effect of the location of the delamination. In fact, the coefficients for different damage degrees (delamination extension) are calculated by AHM, averaging the results of limit cases: the complete separation of the interface and perfect bonding. In other words, AHM (as it is written in the present paper) predicts the same effective coefficients for delaminated composites having the same extension of delamination, but with different damage locations. This problem can be faced by the finite element method (FEM), which can be applied with accuracy to determine effective coefficients for delaminated composites, considering not only the extension of damage but also its location. In addition, another strong result found is that the finite element method, as implemented in this work, is capable of approximating the analytical solution here presented with negligible errors and almost no mesh influence. This shows that the chosen shape functions, along with a careful mesh generation, with no distorted and with good aspect ratio elements, are able to provide a powerful tool for properties prediction in damaged composites. Therefore, the numerical approach can be used for laminates with not only interfaces that show constant properties, but also interfaces that present variable properties as functionally graded material (FGM).

Finally, numerical and analytical approaches studied in the present work can aid to design laminated composites by the prediction of effective properties, considering different delamination extensions. This is very strategic for developing design concepts of different types of laminated composites structures, which can suffer delamination damage in service, in order to suggest good candidates of stacking sequences. Besides, a computer tool could be developed to quantify the influence of delamination in the structure based on data from NDT inspections. Therefore, it would be possible to obtain the residual stiffness, if the delamination follows a pattern of damage. In fact, in a future work, it is worth to highlight that there will be a comparison of numerical and analytical results. Thus, a detailed experimental analysis, employing the NDT via acoustic-ultrasonic technique, is in progress, as well as mechanical tests, and the comparison of the results will be published in future.

Acknowledgements The authors are thankful to Coordination for the Improvement of the Higher Level Personnel (CAPES/ PNPD), National Council for Scientific and Technological Development (CNPq process number: 401170/2014-4 and 310094/2015-1) and PHC Carlos J. Finlay 2018 Project No. 39142TA.

\section{References}

1. Ashari, S.E., Mohammadi, S.: Delamination analysis of composites by new orthotropic bimaterial extended finite element method. Int. J. Numer. Methods Eng. 86, 1507-43 (2011)

2. Aghdam, M.M., Falahatgar, S.R.: Micromechanical modeling of interface damage of metal matrix composites subjected to transverse loading. Comput. Struct. 66, 415-20 (2004)

3. Bonora, N., Ruggiero, A.: Micromechanical modeling of composites with mechanical interface—Part II: damage mechanics assessment. Compos. Sci. Technol. 66, 323-32 (2006)

4. Aghdam, M.M., Falahatgar, S.R., Gorji, M.: Micromechanical consideration of interface damage in fiber reinforced Ti-alloy under various combined loading conditions. Compos. Sci. Technol. 68, 3406-11 (2008)

5. Fang, Q.H., Jin, B., Liu, Y., Liu, Y.W.: Interaction between screw dislocations and inclusions with imperfect interfaces in fiber-reinforced composites. Acta Mech. 203, 113-125 (2009)

6. Mahmoodi, M.J., Aghdam, M.M., Shakeri, M.: Micromechanical modeling of interface damage of metal matrix composites subjected to off-axis loading. Mater. Des. 31, 829-36 (2010)

7. Escarpini Filho, R.S., Marques, S.P.C.: A model for homogenization of linear viscoelastic periodic composite materials with imperfect interface. Lat. Am. J. Solids Struct. 13, 2706-2735 (2016)

8. Almeida, J.H.S., Ribeiro, M.L., Tita, V., Amico, S.C.: Damage and failure in carbon/epoxy filament wound composite tubes under external pressure: experimental and numerical approaches. Mater. Des. 96, 431-38 (2016)

9. Almeida, J.H.S., Ribeiro, M.L., Tita, V., Amico, S.C.: Damage modeling for carbon fiber/epoxy filament wound composite tubes under radial compression. Compos. Struct. 160, 204-210 (2017)

10. Voyiadjis, G.Z., Kattan, P.I.: Introducing damage mechanics templates for the systematic and consistent formulation of holistic material damage models. Acta Mech. 228, 951-990 (2017)

11. Lu, H., Guo, L., Liu, G. et al.: Progressive damage investigation of 2.5D woven composites under quasi-static tension. Acta Mech. (2018). https://doi.org/10.1007/s00707-017-2024-Z 
12. Yam, L.H., Wei, Z., Cheng, L., Wong, W.O.: Numerical analysis of multi-layer composite plates with internal delamination. Comput. Struct. 82, 627-37 (2004)

13. Alfaro, M.V.C., Suiker, A.S.J., Borst, R., Remmers, J.J.C.: Analysis of fracture and delamination in laminates using 3D numerical modelling. Eng. Fract. Mech. 76, 761-80 (2009)

14. Wang, R.G., Zhang, L., Zhang, J., Liu, W.B., He, X.D.: Numerical analysis of delamination buckling and growth in slender laminated composite using cohesive element method. Comput. Mater. Sci. 50, 20-31 (2010)

15. Simon, J.W., Stier, B., Reese, S.: Numerical analysis of layered fiber composites accounting for the onset of delamination. Adv. Eng. Softw. 80, 4-11 (2015)

16. Hirwani, C.K., Panda, S.K., Mahapatra, T.R., Mandal, S.K., Mahapatra, S.S., De, AK.: Delamination effect on flexural responses of layered curved shallow shell panel-experimental and numerical analysis. Int. J. Comput. Methods (2017). https://doi.org/10.1142/S0219876218500275

17. Thai, T.Q., Rabczuk, T., Zhuang, X.: Numerical study for cohesive zone model in delamination analysis based on higher-order B-spline functions. J. Micromech. Mol. Phys. (2017). https://doi.org/10.1142/S2424913017500047

18. Tran, Q.T., Toumi, A., Turatsinze, A.: Delamination of thin bonded cement-based overlays: analytical analysis. Mater. Struct. 44, 43-51 (2011)

19. Song, M.C., Sankar, B.V., Subhash, G., Yen, C.F.: Analysis of mode I delamination of z-pinned composites using a nondimensional analytical model. Composites Part B 43, 1776-84 (2012)

20. Kargarnovin, M.H., Ahmadian, M.T., Jafari-Talookolaei, R.-A., Abedi, M.: Semi-analytical solution for the free vibration analysis of generally laminated composite Timoshenko beams with single delamination. Composites Part B 45, 587-600 (2013)

21. Ojo, S.O., Ismail, S.O., Paggi, M., Dhakal, H.N.: A new analytical critical thrust force model for delamination analysis of laminated composites during drilling operation. Composites Part B 124, 207-17 (2017)

22. Saoudi, J., Zitoune, R., Gururaja, S., Salem, M., Mezleni, S.: Analytical and experimental investigation of the delamination during drilling of composite structures with core drill made of diamond grits: X-ray tomography analysis. J. Compos. Mater. 52, 1281-1294 (2017)

23. Brito-Santana, H., De Medeiros, R., Ferreira, A.J.M., Rodríguez-Ramos, R., Tita, V.: Effective elastic properties of layered composites considering non-uniform imperfect adhesion. Appl. Math. Model. 59, 183-204 (2018)

24. Bensoussan, A., Lions, J.L., Papanicolaou, G.: Asymptotic Analysis for Periodic Structures. North-Holland, New York (1978)

25. Sanchez-Palencia, E.: Non-homogeneous Media and Vibration Theory (Lecture Notes in Physics, 127). Springer Verlag, Berlin (1980)

26. Oleinik, O.A., Panasenko, G.P.: Homogenization and asymptotic expansions for solutions of the elasticity system with rapidly oscillating periodic coefficients. Appl. Anal. 15, 15-32 (1983)

27. Bakhvalov, N.S., Panasenko, G.P.: Homogenization Averaging Processes in Periodic Media. Kluwer Academic Publishers, Dordrecht (1989)

28. Fang, Z., Sun, W., Tzeng, J.T.: Asymptotic homogenization and numerical implementation to predict the effective mechanical properties for electromagnetic composite conductor. J. Compos. Mater. 38, 1371-85 (2004)

29. Hassani, B., Hinton, E.: A review of homogenization and topology optimization II- analytical and numerical solutions of homogenization equations. Comput. Struct. 69, 719-38 (1998)

30. Zienkiewicz, O.C., Taylor, R.L.: The Finite Element Method: The Basis, vol. 1. Butterworth-Heinemann, Oxford (2000)

31. Bezanson, A., Karpinski, S., Shan, V.B., Edelman, A.: Julia: A fast dynamic language for technical computing. arXiv preprint arXiv: 1209.5145 2012;292

32. Hughes, T.J.R.: The Finite Element Method—Linear Static and Dynamic Finite Element Analysis. Prentice-Hall, New Jersey (1987)

33. Geuzaine, C., Remacle, J.F.: Gmsh: a three-dimensional finite element mesh generator with built-in and pre- and postprocessing facilities. Int. J. Numer. Methods Eng. 79, 1309-31 (2009)

34. Tita, V., Carvalho, J., Vandepitte, D.: Failure analysis of low velocity impact on thin composite laminates: experimental and numerical approaches. Compos. Struct. 83, 413-28 (2008)

35. Verbis, J.T., Kattis, S.E., Tsinopoulos, S.V., Polyzos, D.: Wave dispersion and attenuation in fiber composites. Comput. Mech. 27, 244-52 (2001) 